\title{
Application Value of Musculoskeletal Ultrasound in DAA-THA with Conjoined Tendon Repair
}

\author{
Guanbao Li, Pinquan Li, Wei Zhou, Qiuan Chen, Peng Ma, Tongyuan Liu, Hai Tang* \\ The First Department of Hip Joint, Yulin Orthopedic Hospital of Chinese and Western Medicine, Yulin 537000, China \\ *Corresponding author: Hai Tang, tanghai6996@sina.com
}

\begin{abstract}
Objective: To observe the ultrasonographic characteristics of conjoined tendon repair in direct anterior approach for total hip arthroplasty (DAA-THA), and to evaluate the efficacy of musculoskeletal ultrasound in determining the healing after joint tendon repair. Methods: A total of 60 patients who required primary total hip arthroplasty in Yulin Orthopedic Hospital of Chinese and Western Medicine from July 2020 to July 2021 were selected; the patients were divided into two groups, an observation group, group A $(n=30)$, and a control group, group $B(n=30)$, according to different intraoperative methods. There was no significant difference in gender, age, and diagnosis between the two groups. Direct anterior approach was used for both the groups. For group A, the joint capsule and conjoined tendon (superior gemellus, obturator internus, and inferior gemellus) were repaired in situ, whereas for group B, only the joint capsule was repaired in situ, while the conjoined tendon was not repaired. The healing of the tendon was observed. Results: (1) in terms of diagnosis, after conjoined tendon repair, 26 cases in group A showed good tendon continuity, good tension, and a small amount of effusion echo around, three cases showed partial interruption of tendon echo, low echo, or no echo inside with insufficient structural clarity, and a case showed complete interruption; in group B, all 30 cases had continuous interruption, poor tension, tendon retraction, and thickening; the healing rate of group A's conjoined tendon repair was $96.67 \%$; (2) in terms of prognostic assessment, one month after the surgery, the Harris score of group A was significantly higher than that of group $\mathrm{B}(P<0.05)$; however, there was no significant difference in the terms of the Harris score between the two groups 3-6 months after surgery $(P>0.05)$; the effective tension of conjoined tendon and the effective muscle strength of group A were significantly higher than those of group B $(P<0.05)$. Conclusion: Musculoskeletal ultrasound has high diagnostic value in the healing of conjoined tendon and provides dynamic clinical observation after conjoined tendon repair in DAA-THA; it is proven that DAA-THA with conjoined tendon repair on the premise of reconstructing the joint capsule can well restore its tension, enhance its muscle strength, significantly improve early joint stability and joint function, as well as facilitate the rapid recovery of patients.
\end{abstract}

Keywords: Musculoskeletal ultrasound; Direct anterior approach; Total hip arthroplasty; Conjoined tendon; Repair

Publication date: November 2021; Online publication: November 30, 2021

\section{Introduction}

The conjoined tendon comprises of the superior gemellus, internal obturator, and inferior gemellus muscles. It is one of the important components of the small muscle group in the hip joint for external rotation ${ }^{[1]}$. It plays a certain role in the stability and the power used for external rotation of the hip joint. Digital radiography (DR) had a poor effect on soft tissue development. Although magnetic resonance imaging (MRI) has high sensitivity (80\% 97\%) and specificity (93\% 94\%) ${ }^{[2]}$, MRI is an expensive equipment, and its clinical application is limited by its availability and the experience of the operator; in addition, it also takes more time for patients to make an appointment and the cost of waiting and examination is high. Musculoskeletal ultrasound is an emerging ultrasound subspecialty in recent years. It has good temporal and spatial resolution; moreover, it can sensitively and accurately display the state of soft tissues. Therefore, 
it can be used for dynamic comparison and evaluation before tendon release and after repair. More attention has been given to it, and it is now widely used in clinic because of its advantages as it is noninvasive, simple to operate, and results can be obtained rapidly ${ }^{[3]}$. The purpose of this study was to observe the healing after conjoined tendon repair via musculoskeletal ultrasound and other related indexes.

\section{Materials and methods}

\subsection{General information}

A total of 60 patients who needed primary total hip arthroplasty in Yulin Orthopedic Hospital of Chinese and Western Medicine from July 2020 to July 2021 were selected for this study. The patients were divided into two groups: an observation group, group A $(n=30)$, and a control group, group B $(n=30)$, according to different intraoperative methods. There was no significant difference in gender, age, and diagnosis between the two groups; the surgeries were performed by the same senior physician using direct anterior approach. For group A, the joint capsule and conjoined tendon (gemellus superior, obturator internus, and gemellus inferior) were repaired in situ, whereas for group B, only the joint capsule was repaired in situ, while the conjoined tendon was not repaired.

\subsection{Methods, instruments, and equipment}

Siemens's ACUSON Sequoia ultra-high frequency ultrasonic diagnostic instrument was used for inspection, along with 18L6 linear array probe at a frequency of 6-18 MHz, 10L4 linear array probe at a frequency of 4-10 MHz, and 5C1 array probe at a frequency of 5-10 MHz. The inspection methods were carried out as the following steps: fully expose the body surface above the hip joint tendon, and use the ultrasound probe in parallel with the tendon to conduct regular transverse and longitudinal continuous scanning; during the examination, if necessary, the probe can be properly pressurized and scanned repeatedly, or the patient can be instructed to perform internal rotation and external rotation of the hip, combined with the active and passive flexion and extension of the tendon, so as to improve the image quality; then, observe the morphology, internal echo, sliding state, and continuity of the muscle fibers after the conjoined tendon repair; observe the position of the tendon connection, accurately distinguish tendon connection from scar connection, record the adhesion around the tendon, measure the distance of the separation, and compare it with the conjoined tendon before the surgery.

\subsection{Observation indexes}

By observing the tendon after surgery, based on the evaluation of anastomotic stoma and tendon junction, the scar formation can be graded as follows ${ }^{[4]}$ : (1) mild scar, in which the continuity of the tendon is acceptable, the boundary between the tendon and the surrounding tissue is clear or slightly unclear, the thickness of tendon at anastomotic site is less than $5 \mathrm{~mm}$, and the sliding degree of the tendon is $10 \mathrm{~mm}$ or more; (2) severe scar, in which the normal anatomical structure of the tendon has disappeared, the ultrasound shows irregular low echo along with disordered strong echo spots, the boundary between the tendon and the surrounding tissue is unclear, the tendon at the anastomotic site has thickened ( $\geq 5 \mathrm{~mm})$, and the sliding degree of the tendon is less than $10 \mathrm{~mm}$ or none.

Harris scoring system was used to evaluate the hip joint function before and after the surgery. It has four domains: pain, function, deformity, and joint mobility. The full score is 100 , a score $\geq 90$ is excellent, 80 89 is good, and 70 79 is acceptable, and $<70$ is poor.

Manual muscle test (MMT) was used to evaluate the muscle strength of the external rotator muscle. There are 6 grades, and no less than grade 3 is considered as effective muscle strength. 


\section{Results}

\subsection{The state of the tendon after surgery}

The tendons of the patients were observed under ultra-high frequency ultrasound after surgery. For group A, different periods showed different characteristics: 1 2 weeks after surgery, the echo of tendon anastomosis decreased and patchy strong echo can be seen inside with blurred boundaries; 3 4 weeks after surgery, the boundaries of some tendon anastomoses were clear, and fibrous strong echo can be seen inside; about 4 weeks after surgery, the echo of anastomotic stomas was close to normal tendon, reflecting tendon connection (Figure 1). There were 22 cases in total that presented as aforementioned. Among the other 8 cases, there were 6 cases of mild scar connection and 2 cases of severe scar connection. Some anastomotic stomas were significantly thickened, and the boundaries with the surrounding tissues were unclear. The tendon anastomotic site was hypoechoic, the surrounding was irregular hypoechoic, and there was scar connection and a small amount of short line hyperechoic inside (Figure 2).

For group B, the conjoined tendon was interrupted continuously, and the separation was about 0.5-1 $\mathrm{cm}$ (as shown in Figure 3).

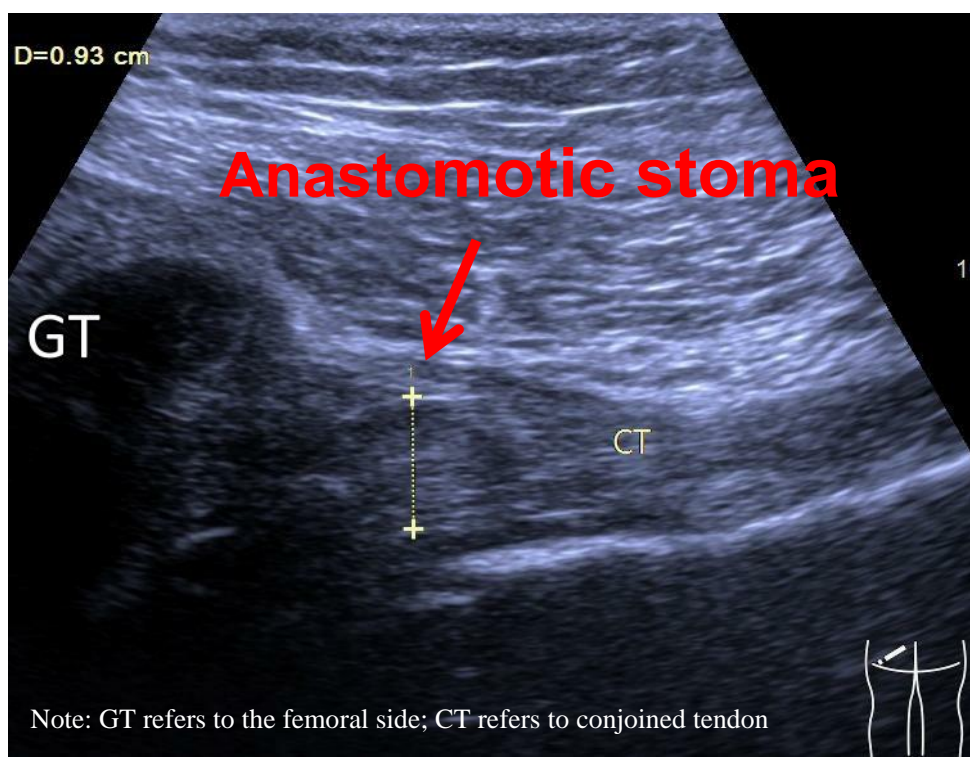

Figure 1. An ultrasound image showing slightly thickened anastomosis and clear boundaries with the surrounding tissue

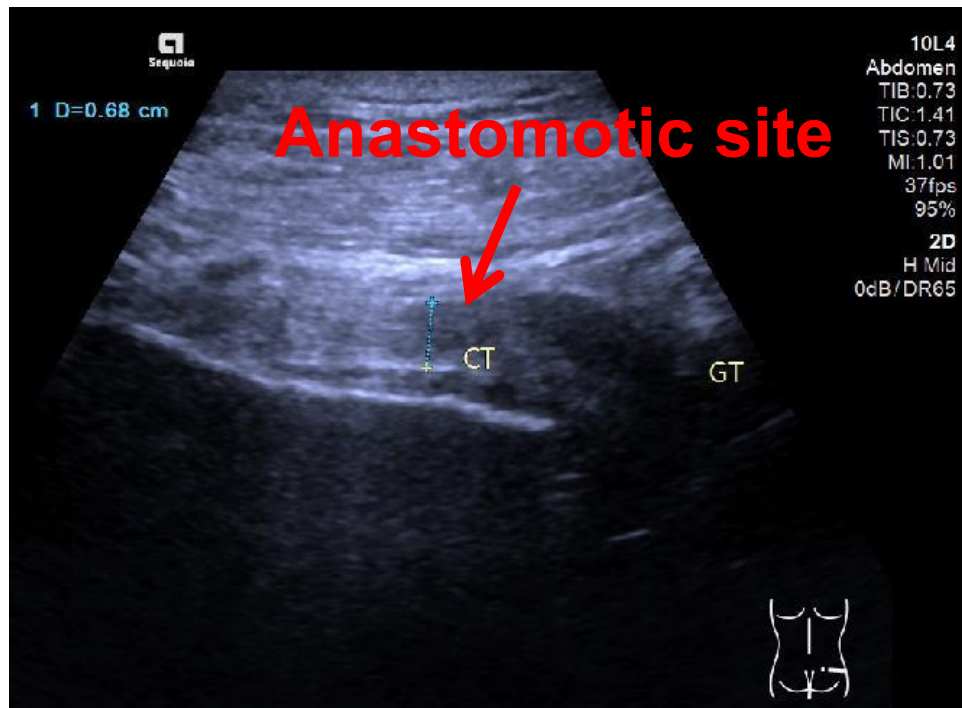

Figure 2. An ultrasound image showing an obviously thickened anastomosis and unclear boundaries with the surrounding tissue 


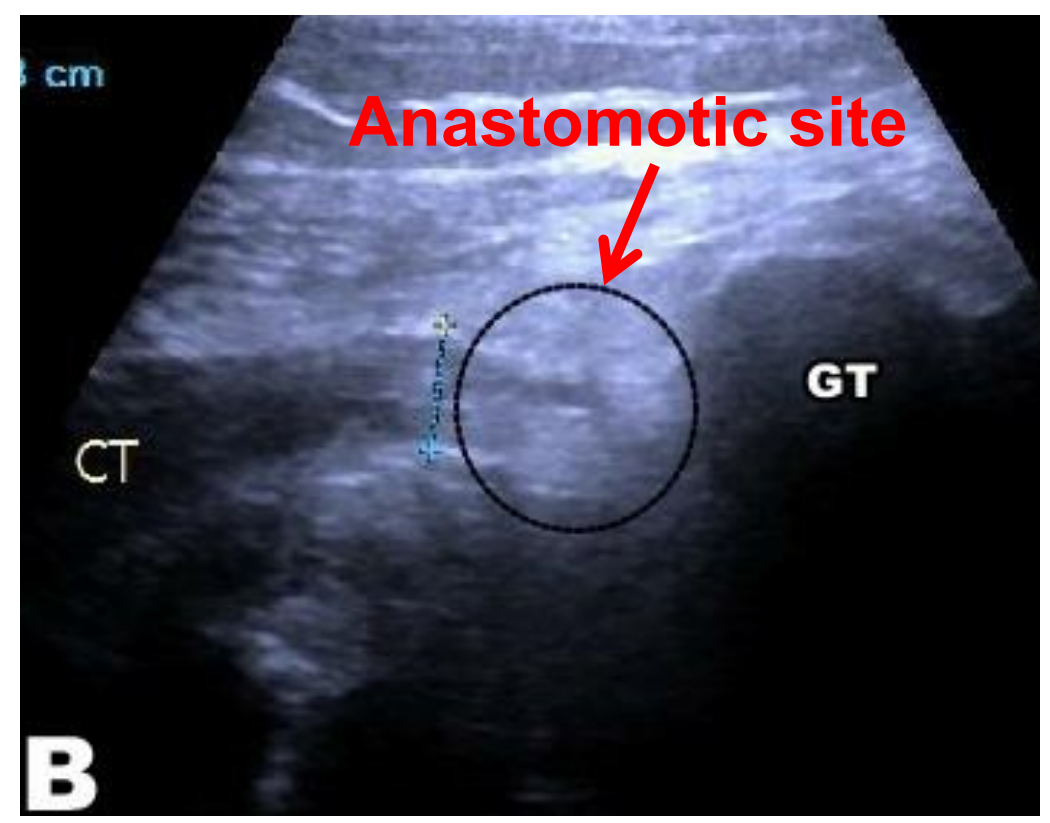

Figure 3. An ultrasound image showing the discontinuity of the conjoined tendon, separated by about $1 \mathrm{~cm}$ with poor tension

\subsection{Harris score, effective muscle strength, and effective tension of conjoined tendon}

One month after surgery, the Harris score of group A was higher than that of group B. There was significant difference between the two groups $(P<0.05)$. Three months after surgery, there was no significant difference in the Harris score between group A and group B $(P>0.05)$. The details are shown in Table 1. The effective muscle strength of group A was significantly higher than that of group $\mathrm{B}(P<0.05)$. The effective tension of conjoined tendon of group A was significantly higher than that of group $\mathrm{B}(P<0.05)$. The details are shown in Table 2.

Table 1. Comparison of Harris scores before and after surgery between the two groups $(\overline{\mathrm{x}} \pm \mathrm{S}$, score/point $)$

\begin{tabular}{lccc}
\hline Group & Before surgery & 1 month after surgery & 3-6 months after surgery \\
\hline Group A $(\mathrm{n}=30)$ & $45.7 \pm 7.0$ & $85.3 \pm 11.3$ & $90.2 \pm 2.4$ \\
Group B $(\mathrm{n}=30)$ & $45.1 \pm 7.2$ & $80.4 \pm 11.7$ & $90.4 \pm 2.9$ \\
$\mathrm{t}$ value & 0.33 & 2.35 & 0.27 \\
$P$ value & $>0.05$ & $<0.05$ & $>0.05$ \\
\hline
\end{tabular}

Table 2. Effective muscle strength and the effective tension of conjoined tendon after surgery

\begin{tabular}{lcc}
\hline Group & Effective muscle strength & Effective tension of conjoined tendon \\
\hline Group A $(\mathrm{n}=30)$ & $28(82.50)$ & $26(86.7)$ \\
Group B $(\mathrm{n}=30)$ & $23(62.50)$ & $21(70.0)$ \\
$\chi^{2}$ value & 4.013 & 4.011 \\
$P$ value & 0.045 & 0.042 \\
\hline
\end{tabular}

\section{Discussion}

In a research ${ }^{[5]}$, it has been confirmed that in joint replacement through DAA, the important factor affecting the lifting of the proximal femur is the conjoined tendon. Loosening the conjoined tendon can raise the proximal femur by about $2.5 \mathrm{~cm}$ to meet the surgical requirements of the femoral side in DAA. The 
difficulty in DAA is that the femoral side cannot be fully lifted, thus having difficulty to meet the surgical requirements of the femoral side. Therefore, tendon release is required for the use of standard handle in DAA. It is known that preserving the anatomical structure of the joint capsule and the minor muscle group for external rotation during total hip arthroplasty would help to restore the soft tissue balance and increase the stability of the hip joint ${ }^{[6]}$. A Japanese scholar once published an article pointing out that the repair of obturator externus tendon is conducive to the prevention of posterior dislocation ${ }^{[7]}$. Therefore, it is of great clinical significance to reconstruct the conjoined tendon in THA through DAA. However, the healing of conjoined tendon after reconstruction cannot be accurately determined only by observing clinical symptoms and signs. On the other hand, radiography and computed tomography (CT) have low resolution in soft tissue. Although MRI can be used to clearly observe soft tissue injuries, it requires time and a high cost; it has strict indication and the conjoined tendon can only be observed statically. However, musculoskeletal ultrasound has been widely used in clinics because of its advantages of being non-invasive, simple to operate, and having no radiation. Repeated examinations can be carried out as it has high repeatability and high resolution for soft tissues ${ }^{[8]}$, which is helpful to observe the healing of conjoined tendon.

(1) Musculoskeletal ultrasound helps in making a definite diagnosis

Tendons are dense connective tissues, mainly comprising of collagen cellulose, which is different from the density of the surrounding muscle and adipose tissue. In addition, the sound velocity and acoustic impedance value are high. Therefore, the state of the tendon can be observed and diagnosed by ultra-high frequency (UHF) ultrasound ${ }^{[9]}$. The results from this study showed that after conjoined tendon repair, 26 cases in group A had good tendon continuity, good tension, and a small amount of effusion echo around; three cases had partial interruption of tendon echo, low echo, or no echo inside with insufficient structural clarity; a case showed complete interruption. In group B, 30 cases had continuous interruption, poor tension, tendon retraction, and thickening. The healing rate of group A, of which the patients underwent conjoined tendon repair was $96.67 \%$, whereas from the results seen in group $\mathrm{B}$, it reflects that the conjoined tendon cannot reconstruct itself.

(2) Musculoskeletal ultrasound helps in evaluating prognosis

One week after surgery, due to the stress reaction of the tissues around the tendon, the anastomotic area would be congested and edematous with lymphocyte infiltration, and ultrahigh frequency ultrasonography would show low echo and blurred boundaries. With the increase of collagen tissues, tendon cells begin to intervene and grow, gradually increasing the echo of anastomotic stoma; patchy or fibrous strong echo can then be seen in the ultrasound. About 4 weeks after surgery, a large number of collagen fibers would proliferate, and the echo of some anastomoses is close to the normal tendon, while other anastomoses with scar connection would show unclear boundaries with the surrounding tissues ${ }^{[10,11]}$. The results from this study showed that eight cases had anastomotic scar connection, with 6 mild cases and 2 severe ones. The Harris score of group A was higher than that of group B. There was significant difference between the two groups $(P<0.05)$. The effective muscle strength of group A was significantly higher than that of group B $(P<0.05)$. The effective tension of conjoined tendon of group A was also significantly higher than that of group B $(P<0.05)$.

In conclusion, musculoskeletal ultrasound has high diagnostic value in the healing of conjoined tendon and provides dynamic clinical observation after conjoined tendon repair in DAA-THA. It has been confirmed that DAA for total hip arthroplasty can restore the tension of conjoined tendon under the premise of reconstructing the structure of the joint capsule, enhance its muscle strength, significantly improve early joint stability and joint function, as well as facilitate rapid recovery. 


\section{Disclosure statement}

The authors declare that there is no conflict of interest.

\section{References}

[1] Suh KT, Park BG, Choi YJ, 2004, A Posterior Approach to Primary Total Hip Arthroplasty with Soft Tissue Repair. Clin Orthop Relat Res, 418(1): 162-167.

[2] Cao D, 2020, Progress in Clinical Research on Ultrasound Diagnosis of Muscle and Tendon Injury due to Various Causes. Chinese General Practice, 23(33): 4274-4278.

[3] Nicholas C, Jennifer L, 2016, Ultrasound Imaging of the Hand and Wrist: Fundamentals and New Perspectives. Musculoskeleta Imaging, 36(4): 43-47.

[4] Zhang X, Cui L, He Y, et al., 2013, Ultrasonic Diagnosis and Grading of Scar Formation After Repair of Flexor Tendon Rupture of Hand. Practical Journal of Medicine \& Pharmacy, 30(7): 613-614.

[5] Zhao G, Zhu R, Jiang S, et al., 2018, Soft Tissue Restrictors of Femoral Elevation in Direct Anterior Approach - An Anatomic Study. Journal of Orthopaedic Surgery and Research, 13: 308.

[6] Li X, Zhou W, Wu H, et al., 2001, Causes and Management of Dislocation after Total Hip Replacement. Orthopedic Journal of China, 8(8): 771-773.

[7] Fujii H, Otani T, Kawaguchi Y, et al., 2020, Preventing Postoperative Prosthetic Joint Dislocation by Repairing Obturator Externus in Total Hip Arthroplasty Performed Via the Posterior Approach. Arthroplasty, 2: 33.

[8] Li C, Zhang D, Wang X, et al., 2020, Diagnostic Value of High frequency Ultrasound in Extensor Finger Tendon Insertion Injury. Chinese Journal of Medical Ultrasound Electronic Edition, 17(7): 697 702.

[9] Zhou W, Li X, Hu H, 2020, Repair of Finger Soft tissue Defect with Free Interosseous Posterior Artery Perforator Flap of Middle and Lower Forearm assisted by High Frequency Ultrasound. Chinese Journal of Microsurgery, 43(3): 285-288.

[10] Collin P, Yoshida M, Delarue A, et al., 2015, Evaluating Postoperative Rotator Cuff Healing: Prospective Comparison of MRI and Ultrasound. Orthop Traumatol Surg Res, 101(Suppl 6): S265S268.

[11] Fang M, Jiang F, Shan Y, 2016, Value of UHF Ultrasound Examination in Diagnosis and Prognosis Evaluation of Finger Tendon Rupture. Anhui Medical and Pharmaceutical Journal, 20(12): 2287-2289. 